\title{
CT findings predict survival of patients with peripheral T cell lymphoma: a preliminary study
}

\author{
Wenbin Yang, Sen Jiang, Jianbang Lin, Yangkang Li \\ Department of Radiology, Cancer Hospital, Shantou University Medical College, Guangdong, China
}

Radiol Oncol 2019; 53(1): 31-38.

Received 28 October 2018

Accepted 19 December 2018

Correspondence to: Yangkang Li, Department of Radiology, Cancer Hospital, Shantou University Medical College, No7, Raoping Rd, Shantou, Guangdong Province 515041, P. R. China. Phone: +86 07548855 5844; Fax: +86 07548856 0352; E-mail: liyangkang@yahoo.com

Disclosure: No potential conflicts of interest were disclosed.

Background. Peripheral T-cell lymphoma (PTCL) is an uncommon disease with poor clinical outcomes. Radiological reports on the survival of patients with PTCL are scarce. The purpose of this study is to investigate the prognostic value of CT findings to predict clinical outcomes in fifty-one patients with histologically proven PTCL.

Patients and methods. The clinical data and CT images of all patients were retrospectively reviewed. CT features including number of involvement sites, lesion size, shape, margin, density, peritumoral invasion, intratumoral necrosis, lymph node involvement, and degree of contrast enhancement were evaluated. Univariate and multiple logistic regression analysis were used to determine the association between the clinical outcome and radiologic factors.

Results. Multiple site involvement, an ill-defined margin with peritumoral invasion, inhomogeneous density, and intratumoral necrosis were found to be associated with poor outcomes in univariate analysis $(P<0.05)$. An ill-defined margin with peritumoral invasion, was identified as an independent risk sign by further multivariate logistic regression analysis $(P<0.05)$. The area under the ROC curve of this $C T$ feature was $0.745(P<0.05)$.

Conclusions. An ill-defined margin with peritumoral invasion was a valuable prognostic factor to predict the worse clinical outcomes in patients with PTCL.

Key words: Iymphoma; peripheral T cell lymphoma; computed tomography; prognosis

\section{Introduction}

Peripheral T-cell lymphoma (PTCL) is an uncommon disease entity more prevalent in Asia than in Western countries and accounts for $5 \%$ to $30 \%$ of all non-Hodgkin lymphomas (NHL). ${ }^{1-3}$ It is a heterogeneous group of clinically aggressive lymphomas including PTCL-not otherwise specified (PTCLNOS), anaplastic large cell lymphoma (ALCL) and angioimmunoblastic T-cell lymphoma (AITL). PTCL is usually associated with poor outcome compared with B-cell lymphomas. ${ }^{4-7}$ Thus, PTCL requires prompt diagnosis and vigilant monitoring of progression since it is a life-threatening disease.

The International Prognostic Index (IPI), which was developed for predicting the clinical course of aggressive lymphomas, is commonly used in PTCL. This system is based on age, performance status, lactate dehydrogenase, stage, and extranodal involvement. Establishing the correct diagnosis and accurately evaluating the extent of tumor involvement are important for patients with PTCL because they directly impact the staging and treatment of the tumor. Therefore, accurately describing the characteristics of PTCL (for example, the tumor size and local extension, lymph node metastases, or extranodal involvement, etc.) is fundamental in clinical imaging practice.

Computed tomography (CT) is usually used for evaluation of the patient who has lymphoma, because it offers the advantage of obtaining information about both the nodal and extranodal 
components of the disease. Hence, it allows for accurate staging of the disease and follow-up of the therapeutic response. Radiologic manifestations about lymphoma have been described in many studies; however, most of them have focused on B-cell lymphoma. Although several reports concerning the imaging characteristics of PTCL have been published ${ }^{8-10}$, these studies usually focused on nodal diseases or conducted on this entity only targeting one extranodal site, for example, the head and neck. Furthermore, the prognostic value of the imaging findings was absent in these studies. The purpose of this study was to evaluate the prognostic value of $\mathrm{CT}$ findings to predict clinical outcomes in patients with PTCL involving both nodal and extranodal sites.

\section{Patients and methods}

\section{Study population and clinical data}

This study was conducted in accordance with the declaration of Helsinki and was approved by the Medical Ethics Committee of Cancer Hospital of Shantou University Medical College. The requirement of informed consent was waived, due to the retrospective nature of the study.

Fifty-one patients with pathologically confirmed PTCL were included in our study through a computerized search of the pathology database between October 2005 and July 2015. The histological diagnosis of PTCL was made by pathologists according to the World Health Organization classification, and all of the biopsy specimens were reviewed through immunohistochemical examination. The patient inclusion criteria were as follows: (a) no patient received any treatment; (b) each patient had complete CT data (unenhanced scan and contrast-enhanced scan of head and neck, chest, and abdominopelvic area); (c) visible tumor on CT. Clinical data collected included the patient's age, gender, tumor location, tumor stage, treatment and outcome.

\section{Image analysis}

CT examinations of 10 patients were performed on a PQ5000 spiral CT scanner (Picker, New York, NY, USA), 41 patients were examined using a GE Brightspeed Elite 16-detector-row scanner (GE Healthcare, Milwaukee, WI, USA). After a series of unenhanced sections, all patients received intravenous bolus injection of contrast medium (Ultravist 300; Bayer Schering Pharma, Berlin-Wedding,
Germany) at a rate of $2.5-3 \mathrm{~mL} / \mathrm{sec}$ and a volume of 75-90 mL. The section thickness of all images of the single spiral CT was $10 \mathrm{~mm}$. For multidetector $\mathrm{CT}$, contiguous axial images and multiplanar reconstructions (MPR) were performed routinely. The section thickness was $5 \mathrm{~mm}$ and reconstruction interval was $1.25 \mathrm{~mm}$.

The qualitative CT findings were reviewed by a consensus between two experienced radiologists (W.B.Y., with 8 years of experience in diagnostic imaging, and S.J., with 12 years of experience in diagnostic imaging). They were aware that the study population had lymphoma, but they were blinded to the pathological type, tumor stage and survival outcome. They evaluated the tumor location, tumor size, margin, shape, density, intratumoral necrosis, peritumoral invasiveness, contrast enhancement pattern, degree of enhancement, and lymph node enlargements. For patients with multiple tumors, the largest tumor was selected as the patient's representative tumor. Tumor size was measured in maximal dimension on the transverse plane. Tumor shape was categorized as round/ oval or irregular. Tumor density was categorized as homogenous or heterogeneous on unenhanced CT images with normal muscle as the standard for comparison. The tumor margin was categorized as well-defined or ill-defined. Areas with reduced or missing contrast enhancement were considered to represent intratumoral necrosis. Peritumoral invasiveness was defined as infiltration of surrounding fat, bony invasion or invasion of the skin. The criteria for vascular invasion were vessel occlusion, focal narrowing, or contour deformity, and more than half of the perimeter in contact with the tumor. ${ }^{11}$ The degree of enhancement was subjectively assessed and categorized as follows: mild, when the enhancement was similar to that of adjacent muscle; moderate, when the enhancement was higher than that of muscle, but lower than that of blood vessels; and marked, when the enhancement was approaching that of blood vessels. Lymph node enlargements were defined as short axis > $1 \mathrm{~cm}$, abnormal round morphology, or central necrosis.

\section{Statistical analysis}

To determine the prognostic value of the CT features, the clinical outcomes of patients were simplified into two categories: poor outcome if lesion progression during therapy or recurrence occurred within 24 months after therapy; good outcome if patients survived for more than 24 months with 
no evidence of recurrence. Recurrence was defined as local (limited to the primary lesion site), distant (disseminates to distant tissues and organs), or both local and distant.

The radiologic variables included for analysis were categorized as follows: involvement site (single or multiple sites), tumor size $(\geq 6.0 \mathrm{~cm}$ or $<6.0$ $\mathrm{cm})$, shape, lesion margin, density, intratumoral necrosis, peritumoral invasion, lymph node involvement, and degree of contrast enhancement.

The $\chi^{2}$ test was applied to compare the frequency of the imaging findings between the poor and good outcome groups in patients. When the radiological signs appeared to be significant in the univariate analysis, multivariate analysis was developed to determine the association between clinical outcomes and individual radiologic variables using logistic regression model.

In the multivariate logistic regression model, variables with a $P$ value less than 0.05 as determined by each univariate analysis were chosen as the independent variables. Odds ratios (OR) as estimates of relative risk with $95 \%$ confidence intervals $(\mathrm{CI})$ were obtained for each risk factor.

The diagnostic performance of each risk factor was established using the area under the receiver operating characteristic (ROC) curve. A two-sided $P$ value of less than 0.05 was considered statistically significant. All statistical tests were performed by using SPSS version 18.0 software (SPSS Inc., Chicago, IL, USA).

\section{Results}

\section{Clinical findings}

As shown in Table 1, the median age of the patients at diagnosis was 47.8 years (ranged 9 to 83 years), and 35 of 51 patients (68.6\%) were below 60 years old. The male to female ratio was 1.7:1. Thirty (58.8\%) patients presented with advanced Ann Arbor stage. According to the histology, there were 27 PTCL-NOS, 15 ALCL (8 anaplastic lymphoma kinase [ALK] positive and 7 ALK negative), and 9 AITL. All the patients received cyclophosphamide, doxorubicin, vincristine, and prednisone (CHOP)based chemotherapy. 11 patients also accepted radiotherapy. Follow-up period ranged from 10 to 115 months (mean, $45.9 \pm 24.6$ months). The lesion showed progression during therapy in 8 patients. Lesion relapse within 24 months after therapy was found in 19 patients, including local relapse in 7, systemic dissemination in 10, and local relapse with dissemination in 2 . These 27 patients were
TABLE 1. Clinical characteristics of 51 patients with PTCL

\begin{tabular}{|c|c|c|}
\hline Characteristics & Number of cases & Percentage(\%) \\
\hline \multicolumn{3}{|l|}{ Gender } \\
\hline Male & 32 & 62.7 \\
\hline Female & 19 & 37.3 \\
\hline Age (y) & \multicolumn{2}{|c|}{$47.8 \pm 19.1$ (range, 9-83) } \\
\hline \multicolumn{3}{|l|}{ Histology } \\
\hline PTCL-NOS & 27 & 52.9 \\
\hline ALCL ALK+ & 8 & 13.7 \\
\hline ALCL ALK- & 7 & 15.7 \\
\hline AITL & 9 & 17.6 \\
\hline \multicolumn{3}{|l|}{ Ann Arbor stage } \\
\hline$|-| \mid$ & 21 & 41.2 \\
\hline III- IV & 30 & 58.8 \\
\hline \multicolumn{3}{|l|}{ Clinical outcome } \\
\hline $\begin{array}{l}\text { Progression or } \\
\text { relapse within } 24 \\
\text { months }\end{array}$ & 27 & 52.9 \\
\hline $\begin{array}{l}\text { No evidence of } \\
\text { relapse within } 24 \\
\text { months }\end{array}$ & 24 & 47.1 \\
\hline
\end{tabular}

$\mathrm{ALCL} A L K+=$ anaplastic large cell lymphoma anaplastic lymphoma kinase positive; $\mathrm{ALCL} A \mathrm{AL}-=$ $\mathrm{ALCL}$ anaplastic lymphoma kinase negative; AITL = angioimmunoblastic T-cell lymphoma; PTCLNOS $=$ PTCL-not otherwise specified

categorized as the poor outcome group. 24 patients survived without any evidence of relapse at least 24 months after therapy, and were classified as the good outcome group.

\section{CT characteristics}

Nodal disease was found in 32 cases (62.7\%). Extranodal involvement was found in 39 cases (76.5\%). The extranodal involvement sites included nasal cavity, paranasal sinus, periorbital area, Waldeyer's ring, parotid gland, lung, liver, spleen, adrenal gland, gastrointestinal tract, breast and musculoskeletal tissue. The size of the tumors ranged from 1.5 to $14.0 \mathrm{~cm}$ (mean, $6.0 \mathrm{~cm}$ ). The shape was depicted as oval $(\mathrm{n}=17)$ or irregular $(n=34)$. The density was described as homogeneous $(n=28)$ and heterogeneous $(n=23)$. A welldefined margin was seen in 29 cases. An ill-defined margin with peritumoral invasion was seen in 22 cases. Intratumoral necrosis was seen in 14 cases. On unenhanced CT images, the density of the solid component of all tumors was 31-58 HU (mean, $45 \mathrm{HU}$ ) which was similar to that of muscle. After contrast medium administration, mild or moderate enhancement was detected in 21 and 30 cases, re- 
TABLE 2. CT findings of 51 patients with PTCL

\begin{tabular}{|c|c|c|}
\hline Characteristics & Number of cases & Percentage(\%) \\
\hline \multicolumn{3}{|l|}{ Involvement site } \\
\hline Single & 27 & 52.9 \\
\hline Multiple & 24 & 47.1 \\
\hline Tumor size $(\mathrm{cm})$ & \multicolumn{2}{|c|}{$6.0 \pm 2.4$ (range, 1.5-14.0) } \\
\hline \multicolumn{3}{|l|}{ Tumor margin } \\
\hline Well-defined & 29 & 56.9 \\
\hline III-defined with peritumoral invasion & 22 & 43.1 \\
\hline \multicolumn{3}{|l|}{ Tumor shape } \\
\hline Round/oval & 17 & 33.3 \\
\hline Irregular & 34 & 66.7 \\
\hline \multicolumn{3}{|l|}{ Tumor density } \\
\hline Homogenous & 28 & 54.9 \\
\hline Heterogeneous & 23 & 45.1 \\
\hline \multicolumn{3}{|l|}{ Intratumoral necrosis } \\
\hline Present & 14 & 27.5 \\
\hline Absent & 37 & 72.5 \\
\hline \multicolumn{3}{|l|}{ Enhancement degree } \\
\hline Mild & 21 & 41.2 \\
\hline Moderate & 30 & 58.8 \\
\hline \multicolumn{3}{|l|}{ Lymph node involvement } \\
\hline Present & 32 & 62.7 \\
\hline Absent & 19 & 37.3 \\
\hline
\end{tabular}

spectively. CT findings of all cases are summarized in Table 2. CT findings of one patient with good clinical outcomes are depicted in Figure 1. CT findings of three patients with poor clinical outcomes are depicted in Figures 2-4.

\section{Prognostic analysis}

The statistical results of univariate analysis are summarized in Table 3. In univariate analysis, four $\mathrm{CT}$ features, including multiple site involvement, an ill-defined margin with peritumoral invasion, intratumoral necrosis, and inhomogeneous density were associated with poor clinical outcomes $(P<$ $0.05)$. Multivariate analysis showed that only one feature, an ill-defined margin with peritumoral invasion, remained a significantly independent predictor of poor clinical outcomes $(P<0.05)$. Further ROC curve analysis showed that the area under the curve of this significant CT feature was $0.745(P<$ $0.05)$, which suggests that the multivariate logistic regression model is a reasonable predictor of clinical outcome (Table 4).

\section{Discussion}

In the present study, an ill-defined margin with peripheral tissue invasion was identified to be an independent risk factor for clinical outcome of patients with PTCL. This CT sign is considered more indicative of squamous cell carcinomas than of NHL, if there is no history of previous treatment or recent infection. For squamous cell carcinoma, an ill-defined margin with peripheral tissue invasion increases the risk of local failure, distant metastases, and decreased survival. ${ }^{10,12}$ To our knowledge, there are only a few reports on the prognostic significance of ill-defined margin and local tumor invasion in NHL, perhaps because they have been considered uncommon findings in malignant lymphoma. Zhou et al. ${ }^{13}$ reported that the lesion margin was an independent risk factor for clinical outcome

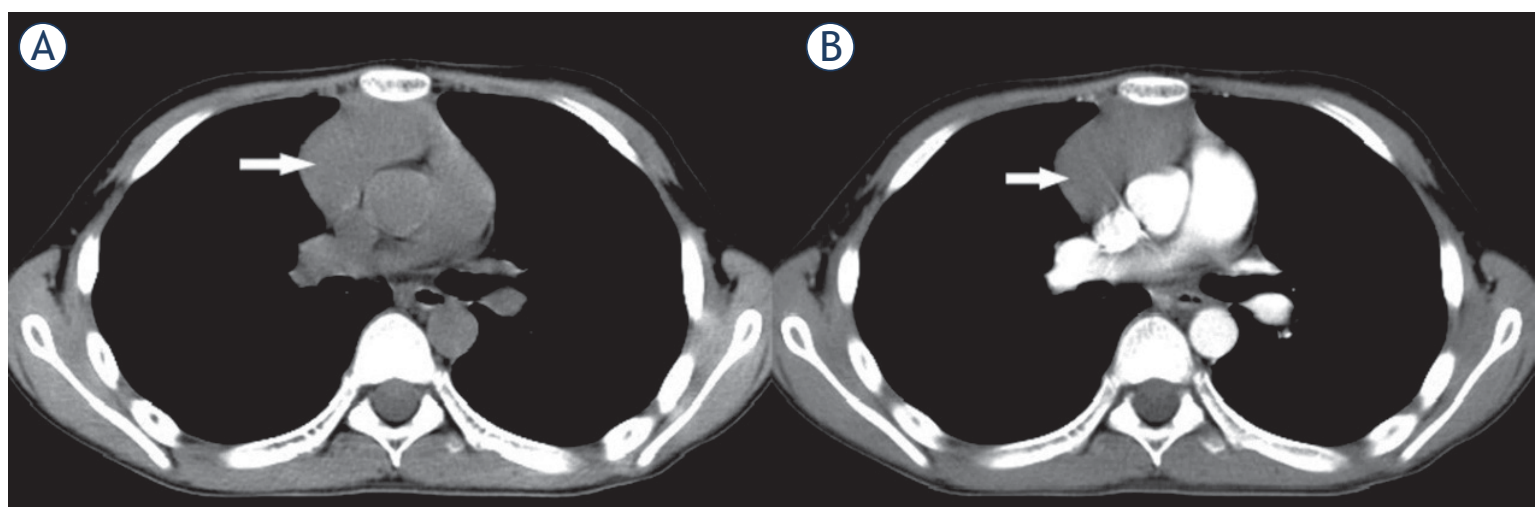

FIGURE 1. A 13-year-old boy with PTCL in the anterior mediastinum. (A) Axial non-contrast CT image shows an oval, well-defined mass with homogeneous density in the anterior mediastinum (white arrow). (B) Contrast-enhanced CT image shows the tumor with homogeneously mild enhancement (white arrow). Tumor recurrence was not noted during the 36-month follow-up period. 
in 59 patients with head and neck NHL. Kim et al. ${ }^{14}$ also reported that local tumor invasion as assessed by CT or MR imaging was a more important prognostic factor than IPI in predicting a low probability of complete remission and lower overall and disease-free survival in extranodal lymphoma. The result of our study was in agreement with previous literatures.

For malignant solid tumors, direct invasion may result in the infiltration of tumor cells to surrounding tissues and neighboring organs. Although a lymphoma is not really considered as a solid tumor, it does form a mass. For aggressive lymphoma subtypes, peritumoral/extracapsular infiltration often occurs. In the present study, the local invasion of PTCL, affected the fat, bone, skin or vessel nearby the tumor and gave the tumor an ill-defined margin on $\mathrm{CT}$ images. Of primary importance in the prognosis of patients with PTCL is the sequence of events leading to the development of tumor cell invasion. In previous studies, extensive deregulation of genes that control functions typically damaged in malignant cells, such as matrix remodeling, cell adhesion, transcription regulation, proliferation, and apoptosis, was found in T-cell or B-cell lymphomas. ${ }^{15-19}$ The complexity of these tumors is represented by 25 to 30 up-regulated cancer genes and several down-regulated tumor suppressors that provide a growth advantage and enhance the ability to invade and disseminate. This might explain the mechanisms of local invasion and distant dissemination of PTCL in our study. The course of tumor invasion entails a series of stages that lead to dissemination and the formation of secondary tumors in distant organs and is, largely, responsible for the mortality and morbidity of PTCL.
TABLE 3. Univariate analyses of CT findings

\begin{tabular}{|c|c|c|c|}
\hline Factor & Category & $\begin{array}{l}\text { Number of } \\
\text { good vs poor } \\
\text { outcomes }\end{array}$ & $P$ value \\
\hline \multirow[t]{3}{*}{ Involvement site } & & & $0.016^{*}$ \\
\hline & Single & $17: 10$ & \\
\hline & Multiple & $7: 17$ & \\
\hline \multirow[t]{3}{*}{ Tumor size } & & & 0.328 \\
\hline & $<6.0 \mathrm{~cm}$ & $14: 13$ & \\
\hline & $\geq 6.0 \mathrm{~cm}$ & $10: 14$ & \\
\hline \multirow[t]{3}{*}{$\begin{array}{l}\text { Ill-defined margin with } \\
\text { peritumoral invasion }\end{array}$} & & & $<0.001^{*}$ \\
\hline & Present & $4: 18$ & \\
\hline & Absent & $20: 9$ & \\
\hline \multirow[t]{3}{*}{ Tumor shape } & & & 0.617 \\
\hline & Round/oval & $8: 9$ & \\
\hline & Irregular & $16: 18$ & \\
\hline \multirow[t]{3}{*}{ Inhomogenous density } & & & $0.001^{*}$ \\
\hline & Present & $5: 18$ & \\
\hline & Absent & $19: 9$ & \\
\hline \multirow[t]{3}{*}{ Intratumoral necrosis } & & & $0.025^{*}$ \\
\hline & Present & $3: 11$ & \\
\hline & Absent & $21: 16$ & \\
\hline \multirow[t]{3}{*}{ Enhancement degree } & & & 0.586 \\
\hline & Mild & 10:11 & \\
\hline & Moderate & $14: 16$ & \\
\hline \multirow[t]{3}{*}{ Lymph node involvement } & & & 0.069 \\
\hline & Present & $12: 20$ & \\
\hline & Absent & $12: 7$ & \\
\hline
\end{tabular}

${ }^{*} P<0.05$

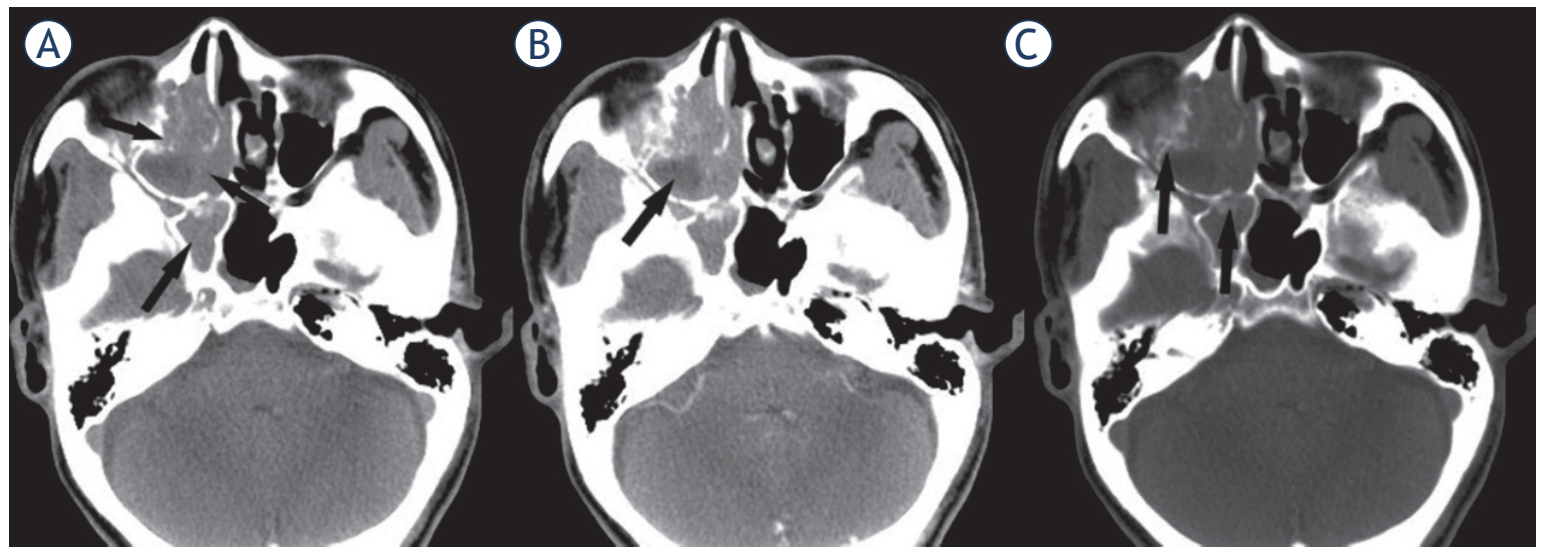

FIGURE 2. A 54-year-old man with PTCL in the sinonasal cavity. (A) Axial non-contrast CT image shows an ill-defined, irregular mass with inhomogeneous density in the right nasal cavity, maxillary and sphenoid sinus (black arrows). (B) Contrast-enhanced CT image shows the tumor with heterogeneously moderate enhancement. Intratumoral necrosis is seen in the mass (black arrow). (C) Bony destruction is detected on non-contrast CT image. The tumor relapsed 11 months after therapy. 


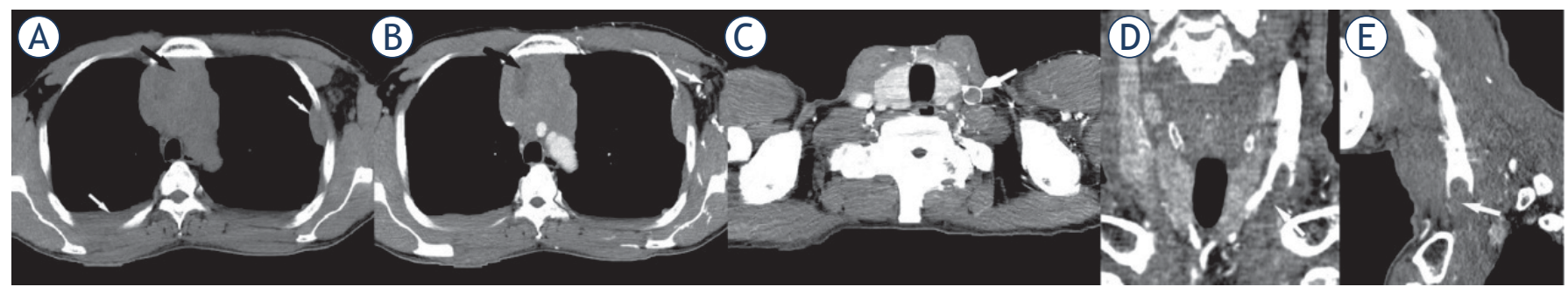

FIGURE 3. A 47-year-old man with PTCL in the anterior mediastinum. (A) Axial non-contrast CT image shows an ill-defined, irregular mass with inhomogeneous density in the anterior mediastinum (black arrow). Multiple pleural metastases are detected (white arrows). (B) Contrast-enhanced CT image shows the tumor with heterogeneously mild enhancement. Intratumoral necrosis is seen in the mass (black arrow). Lymphadenopathy is seen in the left axillary fossa (white arrows). (C, D, E) The left internal jugular vein is invaded by the tumor and vessel occlusion is detected on contrast-enhanced CT image and MPR images (white arrows). Tumor progression was found during therapy. This patient deceased at 3 months after therapy.

TABLE 4. Multivariate analyses of CT findings

\begin{tabular}{lccc}
\hline Factor & Odd ratio & $95 \% \mathrm{Cl}$ & P value \\
\hline Involvement site & 3.499 & $0.766-15.987$ & 0.106 \\
$\begin{array}{l}\text { III-defined margin with peritumoral } \\
\text { invasion }\end{array}$ & 7.749 & $1.567-38.315$ & $0.012^{*}$ \\
Inhomogenous density & 2.356 & $0.324-17.116$ & 0.397 \\
Intratumoral necrosis & 3.157 & $0.253-39.370$ & 0.372 \\
\hline
\end{tabular}

$* P<0.05$ it may suggest the high aggressiveness of the disease, and more aggressive therapy is warranted for such circumstances.

A severely inhomogeneous tumor pattern on CT images was found to be associated with a high malignancy grade in NHL. This CT pattern was also compatible with a poor prognosis in patients treated with chemotherapy. ${ }^{10,20}$ In this study, inhomogeneous density was another radiological sign that was associated with poor survival outcome in univariate analysis. This result was similar to those of previous studies. Although inhomogeneous density can be noted in other diseases, such as epithelial tumor, soft tissue neoplasia or infection, nearly a half of the patients (about $45 \%$ ) with PTCL in our study showed this radiological characteristic. Inhomogeneous density may correspond to asymmetric tumor cell density, intralesional hemorrhage, necrosis or cystic change. This heterogeneity of tumor parenchyma always leads to heterogeneous density on CT images. Intratumoral hemorrhage and cystic change are seldom seen in defined lesion without local tumor invasion. And and understanding the mechanisms concerned in peritumoral invasion may lead to limiting tumor progression and, as a result, to a reduction in mortality for PTCL patients. In our opinion, when PTCL manifests as an ill-defined margin and peritumoral invasion, no matter the nodal disease or the extranodal lesion, patients are significantly more likely to experience poor outcomes than those with a welldefined lesion without local tumor invasion. And

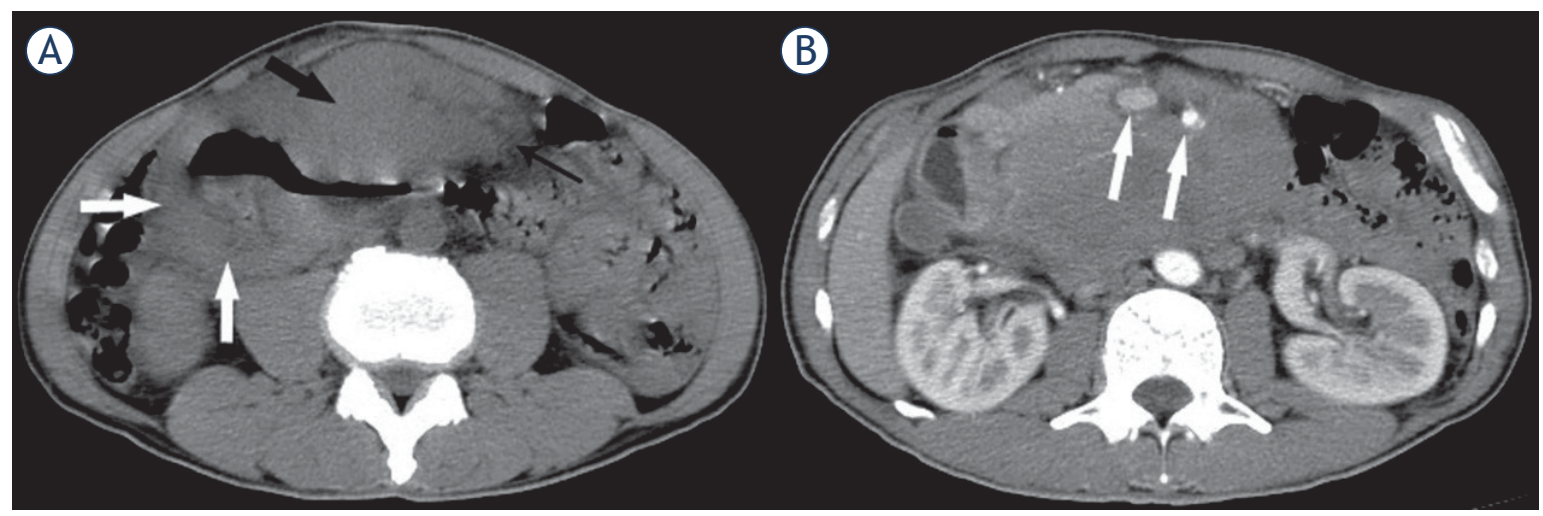

FIGURE 4. A 61-year-old man with PTCL in the deudenum. (A) Axial non-contrast CT image shows the thickening of the duodenal wall (white arrows).The lesion grows in an expansive centripetal fashion (black arrow) with an ill-defined margin and invasion of adjacent fatty tissue (thin black arrow). (B) Contrast-enhanced CT image shows the tumor with heterogeneously mild enhancement. Superior mesenteric artery and vein are encased by the tumor (white arrows). Tumor progression was found during therapy. This patient deceased at 5 months after therapy. 
untreated lymphoma. In our opinion, asymmetric tumor cell density and uneven distribution of blood vessel density in PTCL may contribute to inhomogeneous density on CT images, though the corresponding radiological-pathological studies are absent in our study. A previous study showed that tumor cells of T-cell lymphoma grown under an inhomogeneous cell density, especially under a high cell density environment, become better adapted to survival. They are not only superior in their tumorigenic potential, but are also resistant to the antitumor action of multiple anticancer drugs. ${ }^{21}$ Increased intratumoral vascularity has also been described as a negative prognostic factor in diffuse large B-cell lymphoma (DLBCL) treated with rituximab plus chemotherapy. ${ }^{22}$

Although intratumoral necrosis was not a common feature of NHL, it was found in nearly $27.5 \%$ of patients (14/51) in our study. Three previous studies have investigated the prognostic implications of tumor necrosis at CT or MRI in lymphoma. ${ }^{23-25}$ Hopper et al..$^{23}$ investigated chest CT scans of 76 patients with newly diagnosed Hodgkin lymphoma with mediastinal involvement. Their results showed that the presence of mediastinal necrotic lymph nodes appears to have little prognostic significance in patients with Hodgkin lymphoma. Saito et al. ${ }^{24}$ evaluated CT and MRI scans of 60 patients with different non-Hodgkin lymphoma subtypes for the presence of necrosis in lymph nodes. They found that lymph node necrosis had no significant influence on patients' disease-free survival, but may have a prognostic significance in patients with non-Hodgkin lymphomas. Adams et $a l .{ }^{25}$ studied CT scans of 51 patients with DLBCL for the tumor necrosis in lymph nodes as well as extranodal sites. The findings of their study also indicate the prognostic potential of tumor necrosis at CT in newly diagnosed DLBCL. The result of our study was similar to those of Saito et al. and Adams et al. Univariate analysis showed that intratumoral necrosis was associated with poor clinical outcomes. This may indicate that the underlying PTCL has an aggressive tumor growth. Although intratumoral necrosis was not an independent risk factor by multivariate analysis, it may have a prognostic significance in patients with PTCL.

The results of our study showed that PTCL is likely to involve multiple regions and manifest as a generalized disease. Lee et al. ${ }^{8}$ also found that the most common radiologic feature of PTCL was systemic dissemination, including various organ involvement and generalized lymphadenopathy. In addition, in their series of 581 patients with in- testinal non-Hodgkin lymphoma, Kim et al. ${ }^{26}$ identified that B-cell lymphoma mainly presented as localized disease while T-cell lymphoma involved multiple sites. Three subtypes of T-cell lymphoma including PTCL-NOS, EATL and NK/T lymphoma had a relatively lower response rate and a higher recurrence rate. Although multiple site involvement was not an independent risk factor to predict patients' prognosis in our study, it was associated with poor clinical outcome in univariate analysis. Again, this may suggest that this characteristic is an indicative sign of the aggressive clinical course of PTCL.

In diagnosis, staging, monitoring of treatment and prediction of prognosis in patients with lymphoma, ${ }^{18} \mathrm{~F}$-fluorodeoxyglucose positron emission tomography-computed tomography $\left({ }^{18} \mathrm{~F}-\mathrm{FDG}\right.$ PET/CT) has proved to be a more effective modality than diagnostic CT. ${ }^{27-30}$ Jung et al. ${ }^{31}$ investigated the prognostic accuracy of interim PET/CT in PTCL and derived cutoffs for standardized uptake value (SUV) and metabolic tumor volume (MTV) assessments that were significantly predictive for survival. Cottereau et al..$^{32}$ also reported that higher MTV predicted a poor survival in patients with PTCL. However, in China the limited number of hybrid PET/CT scanners and major financial restrictions also prevent widespread use of this expensive modality. Furthermore, intravenous contrast material administration is usually absent in FDG PET/CT studies and blood vessel invasion by lymphoma may not be clearly depicted. Small disease missed by PET/CT because of the absence of FDG uptake and non-pathological FDG accumulation can also be properly identified by contrast-enhanced CT.

Our study has several limitations, the first of which is its retrospective nature. Second, a detailed pathological study was absent to be correlated to the imaging findings. Therefore, further investigation on large cases with a detailed radiologicalpathological correlation study is needed. Third, different CT equipment and techniques were used. CT examinations of 10 patients were performed on a single spiral CT with a section thickness of $10 \mathrm{~mm}$. Compared with the smaller section thickness, it may affect the quality of the CT images and decrease the probability that more tissue of a given lesion will be captured. Fourth, different histological subtypes of PTCL were involved, and each subtype may have different clinical features and treatment response. However, this problem is simply unavoidable due to the limitations of a retrospective study and the rarity of PTCL, and should not have significantly affected the imaging characteristics studied. 
In conclusion, multiple site involvement, an ill-defined margin with peritumoral invasion, inhomogeneous density, and intratumoral necrosis are relatively significant CT features of PTCL. An ill-defined margin with peritumoral invasion indicates a high risk of unfavorable survival outcome for PTCL. Nevertheless, the interaction between the different clinical and radiologic parameters is complex, and the imaging-based criteria for the assessment of treatment success and outcome is still the subject of ongoing investigations. To reliably evaluate the survival of patients with PTCL, it is important that the imaging examinations be performed according to unified technology and are interpreted according to standardized rules.

\section{References}

1. Anderson JR, Armitage JO, Weisenburger DD. Epidemiology of the nonHodgkin's lymphomas: distributions of the major subtypes differ by geographic locations. Non-Hodgkin's Lymphoma Classification Project. Ann Oncol 1998; 9: 717-20. PMID: 9739436

2. Shea L, Liu J, Cashen A. Prognostic significance of [18F]fluorodeoxyglucosepositron emission tomography in peripheral T-cell lymphoma treated with stem cell transplantation: a retrospective analysis. Leuk Lymphoma 2015 56: 256-9. doi: 10.3109/10428194.2014.914194

3. Park S, Ko YH. Peripheral T cell lymphoma in Asia. Int J Hematol 2014; 99: 227-39. doi: 10.1007/s12185-014-1520-3

4. Yi S, An G, Qi J, Zou D, Zhao Y, Zhang P, et al. The significance of bone marrow involvement in aggressive lymphomas: A retrospective comparison of clinical outcomes between peripheral $T$ cell lymphoma and diffuse large B cell lymphoma in China. Acta Haematol 2010; 124: 239-44. doi: $10.1159 / 000321544$

5. Gisselbrecht C, Gaulard P, Lepage E, Coiffier B, Brière J, Haioun C, et al. Prognostic significance of T-cell phenotype in aggressive non-Hodgkin's lymphomas. Groupe d'Etudes des Lymphomes de I'Adulte (GELA). Blood 1998; 92: 76-82. PMID: 9639502

6. Adams SV, Newcomb PA, Shustov AR. Racial patterns of peripheral T-cell lymphoma incidence and survival in the United States. J Clin Oncol 2016; 34: 963-71. doi: 10.1200/JCO

7. Lim ST, Hee SW, Quek R, Lim LC, Yap SP, Loong EL, et al. Comparative analysis of extra-nodal NK/T-cell lymphoma and peripheral T-cell lymphoma: significant differences in clinical characteristics and prognosis. Eur J Haemato 2008; 80: 55-60. doi: 10.1111/j.1600-0609.2007.00978.x

8. Lee HJ, Im JG, Goo JM, Kim KW, Choi BI, Chang KH, et al. Peripheral T-cell lymphoma: spectrum of imaging findings with clinical and pathologic features. Radiographics 2003; 23: 7-26. doi: 10.1148/rg.231025018

9. Otero $\mathrm{HJ}$, Jagannathan JP, Prevedello LM, Johnston CJ, Ramaiya NH, Van den Abbeele AD, et al. CT and PET/CT findings of T-cell lymphoma. Am Roentgenol 2009; 193: 349-58. doi: 10.2214/AJR.08.1398

10. Choi JW, Kim SS, Kim EY, Heran M. Peripheral T-cell lymphoma in the neck: CT findings of lymph node involvement. Am J Neuroradiol 2006; 27: 107982. PMID: 16687547

11. Lu DS, Reber HA, Krasny RM, Kadell BM, Sayre J. Local staging of pancreatic cancer: criteria for unresectability of major vessels as revealed by pancreatic-phase, thin-section helical CT. Am J Roentgenol 1997; 168: 1439-43. doi: 10.2214/ajr.168.6.9168704

12. Weber AL, Rahemtullah A, Ferry JA. Hodgkin and non-Hodgkin lymphoma of the head and neck: clinical, pathologic, and imaging evaluation. Neuroimaging Clin N Am 2003; 13: 371-92. doi: 10.1016/S1052 5149(03)00039-X

13. Zhou C, Duan X, Lan B, Liao J, Shen J. Prognostic CT and MR imaging features in patients with untreated extranodal non-Hodgkin lymphoma of the head and neck region. Eur Radiol 2015; 25: 3035-42. doi: 10.1007/s00330-0153708-1
14. Kim TM, Park YH, Lee SY, Kim JH, Kim DW, Im SA, et al. Local tumor invasiveness is more predictive of survival than International Prognostic Index in stage I(E)/II(E) extranodal NK/T-cell lymphoma, nasal type. Blood 2005; 106: 3785-90. doi: 10.1182/blood-2005-05-2056

15. Opdam FJ, Kamp M, de Bruijn R, Roos E. Jak kinase activity is required for lymphoma invasion and metastasis. Oncogene 2004; 23: 6647-53. doi: 10.1038/sj.onc.1207887

16. Mahadevan D, Spier C, Della Croce K, Miller S, George B, Riley C, et al. Transcript profiling in peripheral T-cell lymphoma, not otherwise specified, and diffuse large B-cell lymphoma identifies distinct tumor profile signatures. Mol Cancer Ther 2005; 4: 1867-79. doi: 10.1158/1535-7163. MCT-05-0146

17. Pileri SA, Piccaluga PP. New molecular insights into peripheral T cell lymphomas. J Clin Invest 2012; 122: 3448-55. doi: 10.1172/JCI61205

18. Ruddell A, Mezquita P, Brandvold KA, Farr A, Iritani BM. B lymphocytespecific c-Myc expression stimulates early and functional expansion of the vasculature and lymphatics during lymphomagenesis. Am J Pathol 2003; 163: 2233-45. doi: 10.1016/S0002-9440(10)63581-X

19. Ruddell A, Harrell MI, Furuya M, Kirschbaum SB, Iritani BM. B lymphocytes promote lymphogenous metastasis of lymphoma and melanoma. Neoplasia 2011; 13: 748-57. PMID: 21847366

20. Rodriguez M, Rehn SM, Nyman RS, Sundström JC, Ahlström H, Glimelius BL. CT in malignancy grading and prognostic prediction of non-Hodgkin's lymphoma. Acta Radiol 1999; 40: 191-7. PMID: 10080733

21. Singh V, Singh MP, Singh SM. Cell density-dependent alterations in tumorigenic potential of a murine T-cell lymphoma: implication in the evolution of multidrug resistance in tumor cells. Anticancer Drugs 2008; 19: 793-804. doi: 10.1097/CAD.0b013e32830ce4f5

22. Cardesa-Salzmann TM, Colomo L, Gutierrez G, Chan WC, Weisenburger D, Climent F, et al. High microvessel density determines a poor outcome in patients with diffuse large B-cell lymphoma treated with rituximab plus chemotherapy. Haematologica 2011; 96: 996-1001. doi: 10.3324/haematol.2010.037408

23. Hopper KD, Diehl LF, Cole BA, Lynch JC, Meilstrup JW, McCauslin MA. The significance of necrotic mediastinal lymph nodes on CT in patients with newly diagnosed Hodgkin disease. Am J Roentgenol 1990; 155: 267-70. doi: 10.2214/ajr.155.2.2115249

24. Saito A, Takashima S, Takayama F, Kawakami S, Momose M, Matsushita T. Spontaneous extensive necrosis in non-Hodgkin lymphoma: prevalence and clinical significance. J Comput Assist Tomogr 2001; 25: 482-6. PMID: 11351202

25. Adams HJ, de Klerk JM, Fijnheer R, Dubois SV, Nievelstein RA, Kwee TC Prognostic value of tumor necrosis at CT in diffuse large B-cell lymphoma. Eur J Radiol 2015; 84: 372-7. doi: 10.1016/j.ejrad.2014.12.009

26. Kim SJ, Choi CW, Mun YC, Oh SY, Kang HJ, Lee SI, et al. Multicenter retrospective analysis of 581 patients with primary intestinal non-hodgkin lymphoma from the Consortium for Improving Survival of Lymphoma (CISL). BMC Cancer 2011; 11: 321-32. doi: 10.1186/1471-2407-11-321

27. Raanani P, Shasha Y, Perry C, Metser U, Naparstek E, Apter S, et al. Is CT scan still necessary for staging in Hodgkin and non-Hodgkin lymphoma patients in the PET/CT era? Ann Oncol 2006; 17: 117-22. doi: 10.1093/ annonc/mdj024

28. la Fougère $C$, Hundt $W$, Bröckel N, Pfluger T, Haug A, Scher B, et al. Value of $\mathrm{PET} / \mathrm{CT}$ versus PET and CT performed as separate investigations in patients with Hodgkin's disease and non-Hodgkin's lymphoma. Eur J Nucl Med Mol Imaging 2006; 33: 1417-25. doi: 10.1007/s00259-006-0171-x

29. Fueger BJ, Yeom K, Czernin J, Sayre JW, Phelps ME, Allen-Auerbach MS. Comparison of $\mathrm{CT}, \mathrm{PET}$, and PET/CT for staging of patients with indolent non-Hodgkin's lymphoma. Mol Imaging Biol 2009; 11: 269-74. doi: 10.1007/ s11307-009-0200-9

30. Ricard F, Giammarile F, Tychyj-Pinel C, Houzard C, Decullier E, Chateau F, et al. PET-CT and diagnostic CT: the synergy of metabolic and morphological data in onco-haematology. Diagn Interv Imaging. 2014; 95: 55-62. doi: 10.1016/j.diii.2013.07.008

31. Jung SH, Ahn JS, Kim YK, Kweon SS, Min JJ, Bom HS, et al. Prognostic significance of interim PET/CT based on visual, SUV-based, and MTV-based assessment in the treatment of peripheral T-cell lymphoma. BMC Cancer 2015; 15: 198. doi: 10.1186/s12885-015-1193-1

32. Cottereau AS, Becker S, Broussais F, Casasnovas O, Kanoun S, Roques M, et al. Prognostic value of baseline total metabolic tumor volume (TMTVO) measured on FDG-PET/CT in patients with peripheral T-cell lymphoma (PTCL). Ann Oncol 2016; 27: 719-24. doi: 10.1093/annonc/mdw011 